\title{
Spin Effects in Heavy Hybrid Mesons on an Anisotropic Lattice
}

\author{
I.T. Drummond, N.A. Goodman, R.R. Horgan, H.P. Shanahan, L.C. Storoni \\ D.A.M.T.P. \\ Silver Street, Cambridge, England CB3 9EW
}

March 22, 2021

\begin{abstract}
We present a quenched calculation of lowest bottomonium hybrid states on an anisotropic lattice with Landau mean-link tadpole improvement, using the improved NRQCD Hamiltonian. We investigate the quark-spin $s=0,1$ sectors which contain both the non-exotic $1^{--}$and the exotic $1^{-+}$, and demonstrate their degeneracy in the case of the lowest order Hamiltonian. Both states are found at around $1.6 \mathrm{GeV}$ above the $\Upsilon$ ground state. We examine the spin-splitting for several hybrid states $\left(1^{--}, 1^{-+}, 0^{-+}, 2^{-+}\right)$which is due to the $-c_{1} \boldsymbol{\sigma} \cdot \boldsymbol{B} / 2 m_{b}$ term in the NRQCD Hamiltonian, all other terms having negligible effect. The spin splittings are well resolved outside errors and are surprisingly large. We investigate their dependence on $c_{1}$ for several values of $c_{1}$. We calculate one contribution to these splittings using the Born-Oppenheimer picture for hybrids and show that the observed size of the effect is plausibly explained by mixing with hybrid states with more than one constituent gluon.
\end{abstract}

keywords: lattice, gauge, anisotropic, QCD

DAMTP-1999-178 


\section{Introduction}

The spectrum of hybrid mesons provides fresh insight into the structure of QCD. Hybrid mesons are therefore of intense interest, both experimentally and theoretically. In this paper we study heavy hybrids using lattice QCD. The results invite testing in future $B$-factory experiments.

Because of the large mass of the $b$-quark we can apply the NRQCD approach, which has been successful for the $\Upsilon$-system [1, 2, 3], with some confidence. Indeed we expect the quarks in heavy hybrid states to be even more slowly moving than is the case for the $\Upsilon$ itself. We work in the quenched approximation for the gauge fields. However it is unlikely that the use of dynamical gauge configurations would radically change our results.

In order to reconcile computational efficiency with the need for accurate measurement of the relatively large values of hybrid excitations, we use an anisotropic lattice that is spatially coarse but refined in the time direction. The gauge field configurations are determined by the spatially improved gluon field action 画,

$$
S_{n}=-\beta \sum_{x, s>s^{\prime}} \chi^{-1}\left\{\frac{5}{3} \frac{P_{s, s^{\prime}}}{u_{s}^{4}}-\frac{1}{12} \frac{R_{s s, s^{\prime}}}{u_{s}^{6}}-\frac{1}{12} \frac{R_{s^{\prime} s^{\prime}, s}}{u_{s}^{6}}\right\}-\beta \sum_{x, s} \chi\left\{\frac{4}{3} \frac{P_{s, t}}{u_{s}^{2} u_{t}^{2}}-\frac{1}{12} \frac{R_{s s, t}}{u_{s}^{4} u_{t}^{2}}\right\}
$$

where $s, s^{\prime}$ run over spatial links, $P_{s, s^{\prime}}$ and $P_{s, t}$ are $1 \times 1$ plaquettes, $R_{s s, s^{\prime}}$ and $R_{s s, t}$ are $2 \times 1$ plaquettes, $\chi$ is the bare anisotropy.

The tadpole parameters $u_{s}$ and $u_{t}$ are defined to be respectively, the expectation values of the traced spatial and time-like link matrices in the Landau gauge [5]. The actual values are established self-consistently by an appropriate iteration procedure. The choice of Landau mean-link tadpoles is motivated by evidence that their use leads to better continuum behaviour than can be obtained from plaquette tadpoles [6, 0, 8].

The renormalized anisotropy is $\chi_{R}=a_{s} / a_{t}$ where $a_{s}$ and $a_{t}$ are the spatial and time-like lattice spacings respectively. The value of $\chi_{R}$ can be obtained in several different ways. In a separate paper we have determined it by fitting torelon and glueball dispersion relations measured on the lattice and by measuring the heavy quark potential in both the fine and coarse directions [8]. In this paper we use the bare parameters $\beta=$ 1.8 and $\chi=6$ together with the appropriately tuned tadpole parameters $u_{s}=0.7216$ and $u_{t}=0.99208$, with corresponding value $\chi_{R}=5.32(2)$. From the $\Upsilon(1 P-1 S)$ mass gap measured on the lattice we find $a_{t}^{-1}=4503(46) \mathrm{MeV}$ and $a_{s}^{-1}=819(9) \mathrm{MeV}$.

Our calculations are a continuation of previous work [9, 10] in which we investigated the effect of including all terms in the quark NRQCD Hamiltonian up to $O\left(m v^{6}\right)$. However in measuring the heavy hybrids we have found that terms of $O\left(m v^{4}\right)$ and higher have negligible effect on our results. We therefore drop such terms in order to reduce the computational load. This circumstance is consistent with the observation that the heavy quarks move rather slowly in the hybrid state.

The contribution from the first spin dependent term is large however and central to our results. Accordingly we retain this term and the $O\left(a_{s}\right)$ improvement to $D^{2}$, and use the quark Hamiltonian $H=H_{0}+\delta H$, where

$$
\begin{aligned}
H_{0} & =-\frac{D^{2}}{2 m_{b}} \\
\delta H & =-c_{0} \frac{\Delta^{4}}{8 m_{b}^{3}}-c_{1} \frac{g}{2 m_{b}} \boldsymbol{\sigma} \cdot \boldsymbol{B}+O\left(m v^{4}\right) .
\end{aligned}
$$


The $\boldsymbol{B}$-field is constructed from an improved form for $F_{\mu \nu}$ [11]. We take the tree-level value $c_{0}=1$.

The thrust of our investigation is to study the dependence of the hybrid mass spectrum on $c_{1}$. This parameter may be subject to a large renormalization that forces its departure from the tree level value $c_{1}=1$. The states considered are the low-lying magnetic hybrids which are classified as the $1^{--}$, which has quark spin $s=0$, and the $0^{-+}, 1^{-+}, 2^{-+}$, which have $s=1$. The $1^{--}$is non-exotic and is the only one of these states that can be produced directly in $e^{+} e^{-}$collisions. Of the others, the $1^{-+}$is exotic.

In section 2 we outline the NRQCD approach to calculating heavy hybrid states, and discuss the operators and smearing used. In section 5 we discuss finite-size effects and present the results from our simulation. In section 6 we discuss the bag model calculation of spin effects and inter-state mixing in the Born-Oppenheimer approximation. In section 7 we present our discussion and draw our conclusions.

\section{The NRQCD approach}

The NRQCD approach to computing the heavy hybrid propagator is well known The Euclidean time evolution equation for the quark propagator $G(\mathbf{x}, t ; \mathbf{y})$ is computed according to the prescription:

$$
G(\mathbf{x}, t+1 ; \mathbf{y})=\left(1-\frac{a_{t} H_{0}}{2 n}\right)^{n} U_{t}^{\dagger}(\mathbf{x})\left(1-\frac{a_{t} H_{0}}{2 n}\right)^{n}\left(1-a_{t} \delta H\right) G(\mathbf{x}, t ; \mathbf{y}) \quad t>0,
$$

where $H_{0}$ and $\delta H$ are given in eq(1.2) and $n=2$ for our simulations. The quark propagator is then determined by its initial distribution, $G(\mathbf{x}, t=0 ; \mathbf{y})=\Gamma(\mathbf{x}, \mathbf{y})$. The choice of initial distribution is governed by the use to be made of the propagator. We use propagators computed from the following in initial distributions:

$$
\begin{aligned}
\Gamma^{L}(\mathbf{x}, \mathbf{y}) & =\delta^{(3)}(\mathbf{x}, \mathbf{y}) \\
\Gamma^{S}(\mathbf{x}, \mathbf{y}) & =\left(1+\frac{l^{2} D^{2}}{4 m}\right)^{m} \delta^{(3)}(\mathbf{x}, \mathbf{y}), \\
\Gamma_{i}^{P}(\mathbf{x}, \mathbf{y}) & =\left(1+\frac{l^{2} D^{2}}{4 m}\right)^{m} D_{i} \delta^{(3)}(\mathbf{x}, \mathbf{y}), \\
\Gamma_{i}^{H}(\mathbf{x}, \mathbf{y}) & =D^{2}\left(1+\frac{l^{2} D^{2}}{4 m}\right)^{m} B_{i} \delta^{(3)}(\mathbf{x}, \mathbf{y}),
\end{aligned}
$$

where $D_{i}$ is a (discrete) covariant derivative. The propagator resulting from the local source, $\Gamma^{L}$, is combined with the each of the other smeared propagators, after hermitian conjugation, and traced with appropriate spin matrices to create the correlators for the set of operators listed in Table 1 .

The correlators appropriate to hybrid states are computed from $\Gamma_{i}^{H}$. An analysis of hybrid states in the Born-Oppenheimer approximation indicates that the quark and anti-quark tend to remain apart from one another because they experience an angular momentum barrier and a color repulsion (the $q \bar{q}$ system is in a color octet). The "donut" smearing incorporated in $\Gamma^{H}$ through the application of a final $D^{2}$, was successfully invoked in order to improve the overlap of the operators with the widely separated $q \bar{q}$ system in the hybrid. 
The forms of the operators we use to interpolate the magnetic hybrid states are shown in Table 11.

\begin{tabular}{|c|c|c|c|c|c|}
\hline & State & $\mathrm{s}$ & $\mathrm{l}$ & $\mathrm{j}$ & $J^{P C}$ \\
\hline spin-0 S-wave & $\bar{q} q$ & 0 & 0 & 0 & $0^{-+}$ \\
\hline spin-1 S-wave & $\bar{q} \sigma_{i} q$ & 1 & 0 & 0 & $1^{--}$ \\
\hline spin-0 P-wave & $\bar{q} D_{i} q$ & 0 & 1 & 0 & $1^{+-}$ \\
\hline \multirow{3}{*}{ spin-1 P-wave } & $\bar{q} \sigma_{i} D_{i} q$ & 1 & 1 & 0 & $0^{++}$ \\
& $\bar{q} \epsilon_{i j k} \sigma_{j} D_{k} q$ & 1 & 1 & 0 & $1^{++}$ \\
& $\bar{q}\left(\sigma_{i} D_{j}+\sigma_{j} D_{i}-\frac{2}{3} \delta_{i j} \sigma_{k} D_{k}\right) q$ & 1 & 1 & 0 & $2^{++}$ \\
\hline spin-0 Hybrid & $\bar{q} B_{i} q$ & 0 & 0 & 1 & $1^{--}$ \\
\hline \multirow{3}{*}{ spin-1 Hybrid } & $\bar{q} \sigma_{i} B_{i} q$ & 1 & 0 & 1 & $0^{-+}$ \\
& $\bar{q} \epsilon_{i j k} \sigma_{j} B_{k} q$ & 1 & 0 & 1 & $1^{-+}$ \\
& $\bar{q}\left(\sigma_{i} B_{j}+\sigma_{j} B_{i}-\frac{2}{3} \delta_{i j} \sigma_{k} B_{k}\right) q$ & 1 & 0 & 1 & $2^{-+}$ \\
\hline
\end{tabular}

Table 1: The forms of the operators used to interpolate the magnetic hybrid states we study and their $J^{P C}$ classification. Here $l$ and $s$ are the $Q \bar{Q}$ angular momentum and spin, and $j$ is the gluon angular momentum.

The charge conjugation $(C)$ and parity $(P)$ of the quarkonia and hybrids, satisfy

$$
\begin{aligned}
& \text { pure magnetic electric } \\
& \text { quarkonium hybrids hybrids } \\
& C=(-1)^{l+s} \quad(-1)^{l+s+1} \quad(-1)^{l+s+1} \\
& P=\quad(-1)^{l+1} \quad(-1)^{l+j} \quad(-1)^{l+j+1}
\end{aligned}
$$

where $l$ and $s$ are the $\bar{q} q$ angular momentum and spin and $j$ is the gluon angular momentum. The S,P and some of the hybrids are listed in Table 1.

\section{Computational details}

In the source and sink operators (Table 1) all links were smeared using covariant smoothing in a manner similar to that described in eq(2.1) with an appropriately defined $D^{2}$. The $\boldsymbol{B}$ fields were computed from the smeared links and were themselves subsequently smeared in a similar manner. The range for the smearings was typically $l=2$ on gauge links and $l=3$ on the quark source. The effectiveness of the smearing was confirmed by a reduction in statistical errors by a factor of three or more. To reduce statistical errors to within $1 \%$ we generally required between 40,000 and 60,000 independent correlators. We used a 50 sweep Cabbibo-Marinari thermalisation to generate the initial gauge fields, and then employed 10 sweeps between measurements.

The computations were performed on the Hitachi SR2201 of the Cambridge High Performance Computing Facility and at University of Tokyo Computer Centre. They were farmed across 64 processors each of which returned statistically independent results that were used to construct the full covariance matrix. In the standard way we performed a correlated fit to the correlation functions of the relevant operators in order to determine the masses of the quarkonium and hybrid excitations. We confirmed that 10 sweeps between measurements was sufficient for decorrelation. 
The timings for the calculation was such that for the hybrids an 8 hour job produces about 80 correlators per processor for the $6^{3} \times 36$ lattice, about 25 for the $8^{3} \times 48$ and 5 for the $10^{3} \times 60$.

\section{Finite size effects}

We first examined the sensitivity of the calculation to finite size effects. In Table 2 we show the mass gap from the $\Upsilon(1 S)$ to the $1^{--}$hybrid (without spin effects) measured by us on lattices $L^{3} \times T$ for a range of values of $L$. Only when $L$ is reduced down to 4 is there any detectable effect from the finite spatial lattice volume. This is consistent with the results of Manke et al [9, 10] .

Since a hybrid is typically $2-3 \mathrm{GeV}^{-1}$ [12] across, its size is $\sim 4 a_{s}$. For $L=6$ it covers a substantial part of the lattice volume. It is surprising therefore that finite size effects are so small since we might expect that a quark would react more strongly to the nearby image of an anti-quark across the boundary of the spatial lattice than to the more distant anti-quark within the the spatial volume. However a strong effect depends on a transition in which the gauge field flux tube switches from the original quark anti-quark linkage to one crossing the spatial boundary. The corresponding transition amplitude involves the expectation value of a closed Polyakov loop that encircles the torus. This vanishes in the confining phase because of the $Z_{3}$ centre symmetry of the action. We argue therefore that our results are consistent with a picture in which the major interaction across the boundary of the spatial box is a weak Van der Waals-type force.

There are other re-linking transitions, in the case of $S U(3)$, involving the multiple wrapping of flux lines round the torus, that do not vanish. However we expect them to be suppressed because of the high energy of the associated flux tubes.

For the rest of our investigation we worked with $L=6$ as the most economical choice of lattice consistent with negligible finite size effects.

\section{Results}

We use as an energy baseline the spin averaged $\Upsilon(1 S)$ state. Table 3 shows our results for the dependence of this baseline on $c_{1}$. They are consistent, with quadratic behaviour in $c_{1}$ reflecting the fact that the shift is due to a quark mass renormalization.

Since the (spin averaged) $1 S-1 P$ mass difference $\Delta M_{P S}$ in lattice units is known to be insensitive to the quark mass $m_{b}$ we use it to determine the temporal lattice spacing in physical units. From the assignment $\Delta M_{P S}=440 \mathrm{MeV}$ we infer that $a_{t}^{-1}=4503(46) \mathrm{MeV}$. From other work [8] we know that $\chi_{R}=5.32(2)$ yielding $a_{s}^{-1}=819(9) \mathrm{MeV}$. We determine $m_{b}$ by measuring the mass of the $\Upsilon\left(1^{3} S\right)$ state from its dispersion relation and then requiring it to be consistent with the mass measured directly. We find $m_{b}=4.75 \mathrm{GeV}$.

Table 10 shows our results for the masses of the hybrid states. We also show here the behaviour of the mass with variation of the $c_{1}$ co-efficient in $\delta H$, which controls the magnitude of the $\boldsymbol{\sigma} . \mathbf{B}$ term. These results are illustrated in Fig. 11.

For each choice of $c_{1}$ (defined in eq(1.2)) we compute the $s=0,1, l=0$ quarkonium correlators and the hybrid correlators for the states listed in Table 1. Although, in principle, we could consider a matrix of source-sink operator pairs and fit the corresponding matrix correlator, we found it sufficient to consider the one correlator for 


\begin{tabular}{|c|c|c|c|c|}
\hline$(\mathrm{L}, \mathrm{T})$ & $(4,50)$ & $(6,36)$ & $(8,48)$ & $(10,60)$ \\
\hline$\left(M_{H}-M_{\Upsilon}\right) a_{t}$ & $0.281(7)$ & $0.244(7)$ & $0.244(9)$ & $0.247(9)$ \\
\hline
\end{tabular}

Table 2: Dependence of the mass, $M_{H}$, of the $1^{--}$hybrid state on the finite size, $L$. In all cases $c_{1}=0, a_{t}^{-1}=4503(46) \mathrm{MeV}$.

\begin{tabular}{|c|c|c|c|}
\hline$c_{1}$ & $1 S_{3}^{1}$ & $1 S_{1}^{0}$ & $\langle 1 S\rangle_{\text {spin }}$ \\
\hline 0 & $0.1237(7)$ & $0.1246(6)$ & $0.1239(5)$ \\
\hline 1 & $0.1196(4)$ & $0.1143(5)$ & $0.1183(3)$ \\
\hline 2 & $0.1054(7)$ & $0.086(3)$ & $0.1005(3)$ \\
\hline
\end{tabular}

Table 3: Mass of the $1 S_{3}^{1}, 1 S_{1}^{0}$ and the spin-averaged value for different values of $c_{1}$.

which both source and sink were smoothed as described above. We found that the $S$-wave quarkonium correlators were well fitted with two-exponentials over the range (in lattice units) $10 \leq t \leq 50$. The hybrid states are much heavier and the signal for the propagator was too noisy for $t>15$ but we obtained excellent fits with a single exponential for typically $t>5$. An representative effective mass plot for the $1-+$ for $c_{1}=-1$ is shown in Fig. 2 2 which has a plateau extending from $t=4-10$. The dashed lines shown the upper and lower error bounds from the fit.

The $1^{--}$hybrid is of particular interest since it will appear as an intermediate state in $e^{-} e^{+}$scattering at $B$-meson factories. It is noteworthy that its excitation energy above the ground state is unchanged by the spin interaction independently of its strength. The absence of a term $O\left(c_{1}\right)$ implies that there is no direct energy shift induced by the spin term in the Hamiltonian and the absence of a term $O\left(c_{1}^{2}\right)$ implies that there is no mixing with quarkonia even though this is possible on the basis of quantum numbers. We explain below why we feel that this result is compatible with the bag model of hybrid states. Our prediction for its mass is $1644(17) \mathrm{MeV}$ above the ground state. This is compatible with, if somewhat higher than, the value from some other studies [9, 10, 13, 14]. It would be of great interest to observe experimentally a state at this energy level.

In contrast the masses of the spin one states are strongly affected by the spin term in the NRQCD Hamiltonian. Of these the $1^{-+}$is exotic and cannot mix with quarkonium states. For this state we have investigated the dependence of its mass on $c_{1}$ for both positive (physical) and negative (unphysical) values. This allows us to disentangle contributions that are linear and quadratic in $c_{1}$. From Table we see that the effect of the spin term is large. For the tree level value $c_{1}=1$ the $1^{-+}$is depressed by $\sim 130$ $\mathrm{MeV}$. A large renormalization of $c_{1}$ because of radiative corrections means that $c_{1}=2$ is a possibility, a value which causes the $1_{-+}$to be depressed by almost $300 \mathrm{MeV}$ to $1265(26) \mathrm{MeV}$ above the spin-averaged $1 S Q \bar{Q}$ state. A simple fit to the $1^{-+}$masses is

$$
a_{t}\left(E_{H}-E_{Q \bar{Q}}\right)=0.369(4)-0.026(2) c_{1}-0.09(1) c_{1}^{2},
$$

with $\chi^{2}=0.3$. These effects are unexpectedly large since spin effects depend on the quark velocity which is low in hybrid states.

The non-exotic $0^{-+}$and $2^{-+}$states can mix with quarkonium states and, in principle, there can be contamination of the signal which would make these states hard to pick out in the simulation. However, as in the case of the $1^{--}$we find no such problem. 


\begin{tabular}{|c|c|c|c|c|c|}
\hline State & $c_{1}$ & $\chi^{2} /$ d.o.f & $a_{t} E_{H}$ & $a_{t}\left(E_{H}-E_{Q \bar{Q}}\right)$ & $E_{H}-E_{Q \bar{Q}} \mathrm{MeV}$ \\
\hline $0^{-+}$ & 1 & 0.91 & $0.444(9)$ & $0.331(9)$ & $1490(43)$ \\
& 2 & 0.34 & $0.32(1)$ & $0.22(1)$ & $991(46)$ \\
\hline $1^{--}$ & 0 & 0.98 & $0.491(5)$ & $0.367(5)$ & $1653(27)$ \\
& 1 & 0.48 & $0.4815(43)$ & $0.3632(43)$ & $1635(25)$ \\
& 2 & 0.85 & $0.4702(65)$ & $0.3648(65)$ & $1643(33)$ \\
\hline & -2 & 0.9 & $0.486(6)$ & $0.385(6)$ & $1734(32)$ \\
& -1 & 1.02 & $0.505(5)$ & $0.387(5)$ & $1743(28)$ \\
$1^{-+}$ & 0 & 0.26 & $0.4887(50)$ & $0.365(5)$ & $1644(27)$ \\
& 1 & 0.90 & $0.4540(60)$ & $0.336(6)$ & $1513(31)$ \\
& 2 & 0.5 & $0.382(5)$ & $0.281(5)$ & $1265(26)$ \\
\hline $2^{-+}$ & 1 & 0.63 & $0.516(6)$ & $.398(6)$ & $1792(32)$ \\
& 2 & 0.96 & $0.488(7)$ & $.388(7)$ & $1747(36)$ \\
\hline
\end{tabular}

Table 4: Mass of the hybrid states with variation of the spin coupling magnitude, accurate to $O\left(m v^{6}\right)$ with $\beta=1.8, a m_{b}=4.75, a_{t}^{-1}=4.503(46) \mathrm{GeV}$.

On the basis of a bag model calculation this mixing is indeed found to be very small and certainly at a level of less than $1 \%$ in our simulations. The $0^{-+}$is depressed even more than the $1^{-+}$and for $c_{1}=2$ is only $\sim 1000 \mathrm{MeV}$ above the $Q \bar{Q}$ ground state. The $2^{-+}$rises for $c_{1}>0$ although the magnitude of the shift is not as great as that for the other states. This is compatible with a change of sign of the coefficient of $c_{1}$ in the fit eq(5.1) which causes this term to compete with the quadratic contribution. We shall argue in the next section that at least a part of the coefficient of the term linear in $c_{1}$ is proportional to $\boldsymbol{L} \cdot \mathbf{s}$ which does indeed have this qualitative behaviour. We have not investigated the $0^{-+}, 2^{-+}$for $c_{1} \leq 0$.

\section{Born-Oppenheimer Model}

Hasenfratz et. al [12] and Kuti and Morningstar [14 have stressed the usefulness of a Born-Oppenheimer picture of hybrid states in the bag model of hadrons. We suggest that our results can be consistently interpreted in this approach. We investigate the effect on mass shifts of a gluon exchanged between the quark and the anti-quark, and the mixing effect induced by transitions to a two-gluon state. We find the former to be very small while the latter can provide a sizeable contribution that goes some way to explaining our observations on the mass shifts of the hybrid states. We also estimate the mixing of the hybrid and quarkonia states and find this to be negligibly small for all reasonable values of $c_{1}$.

For simplicity we assume that the bag is spherical. The wavefunction for a hybrid of total orbital angular momentum $(L, m)$ in the $\mathrm{BO}$ picture is

$$
\Psi_{L m}^{\Lambda}\left(\mathbf{r}, \boldsymbol{r}_{g}^{\prime}\right)=f_{j \Lambda}^{L}(r) D_{m \Lambda}^{* L}(\Omega) \boldsymbol{A}_{j \Lambda}^{\lambda}\left(\boldsymbol{r}_{g}^{\prime}\right)
$$

where $\mathbf{r}$ is the quark-antiquark separation vector with orientation $\Omega=(\theta, \phi), \boldsymbol{r}_{g}^{\prime}$ is the gluon position vector in the body frame, $j$ is the angular momentum of the gluon mode with projection $\Lambda$ onto the body-fixed z-axis, and $\lambda=0,1$ label TM and TE modes, respectively. The total angular momentum is $\boldsymbol{J}=\boldsymbol{L}+\mathbf{s}$. States of definite parity are 
given by

$$
\Psi_{L m}^{|\Lambda| \eta}=\frac{1}{\sqrt{2}}\left(\Psi_{L m}^{|\Lambda|}+\eta \Psi_{L m}^{-|\Lambda|}\right) .
$$

The parity $(P)$ and charge-conjugation $(C)$ are given by

$$
P=\eta(-1)^{L+\lambda+1}, \quad C=\eta(-1)^{L+j+s+1} .
$$

Note, that these assignments do not agree with those quoted in the original reference [12], but they do agree with Le Yaouanc et. al [15] and Rakow [16] who remarked on the discrepancy. We have carefully checked that these formulae are correct. The important point is that the $P C$ classification is independent of $\Lambda$. States with $|\Lambda|=0,1,2, \ldots$ are labelled respectively by $\Sigma, \Pi, \Delta, \ldots$. The lowest lying states include those with $L=1, j=1$ on which we shall concentrate. There are both $\Sigma$ and $\Pi$ states with the same $J^{P C}$ quantum numbers, namely, $1^{--}$with $s=0$ and $0^{-+}, 1^{-+}, 2^{-+}$with $s=1$.

There are two simple processes resulting from the $\boldsymbol{\sigma} . \boldsymbol{B}$ interaction, gluon exchange between the quarks, and gluon exchange between the hybrid gluon and one or other of the quarks. The former is a weak effect because it involves the behaviour of the $Q \bar{Q}$ wavefunction near the origin where it is suppressed both by the angular momentum barrier and a repulsive potential due to the octet nature of the $Q \bar{Q}$ state. An estimate yields a result of order $1 \mathrm{MeV}$ or less.

The large effect is due to gluon exchange between the constituent gluon and the quarks. This effect is large for two reasons: the colour factors are large and it depends on the quark wavefunction in the bulk and not on its value at the origin. We outline the calculation below and follow the formalism of Close and Horgan [17, 18] and Barnes, Close and Monaghan [19, 20]. The latter authors present a calculation of this spin effect in the MIT bag model and we do not reproduce all the details here. The calculation is for the contribution of second-order perturbation theory to the mass shift. The intermediate states are summed over and, in our calculation, are BO states with two constituent gluons. We examine the contribution from one intermediate state with $j_{1}=j_{2}=1, \lambda_{1}=\lambda_{2}=1$. the diagram for this process is shown in Fig. 3 and corresponds to the exchange of a $j=1$ electric gluon. In this case, it turns out that $L^{\prime}=L, s^{\prime}=s$ and $\Lambda_{1}+\Lambda_{2}=\Lambda$, where $\Lambda$ labels the initial state gluon and $\Lambda_{1}, \Lambda_{2}$ label the intermediate state gluons as shown in Fig. 3. Since $\Delta M_{H}$ also does not depend on the sign of $\Lambda$ we consider $\Lambda=0,1$ which correspond to the $\Sigma$ and $\Pi$ states, respectively. The bag is taken to be spherical of radius $R_{B}$. The interaction Hamiltonian and the expressions for the gluon modes, $\boldsymbol{A}_{j \Lambda}^{\lambda}$, and associated $\boldsymbol{B}$ fields are given in 19. The field operators contributing to the mass shift are expanded on this mode basis using the usual creation and annihilation operators. The two-gluon intermediate state can be written in the BO approximation in a form similar to that in eqn. (6.1). After some manipulations of the $D$-matrices involved and some algebra the mass shift takes the form:

$$
\Delta M_{H}=\frac{i C c_{1} \alpha_{s}}{m_{b} R_{B}^{2}}(-1)^{\Lambda} \boldsymbol{e}_{\Lambda} \cdot\left(\boldsymbol{e}_{\Lambda_{1}}^{*} \wedge \boldsymbol{e}_{\Lambda_{2}}^{*}\right) \boldsymbol{L} \cdot \mathbf{s},
$$

where the $\boldsymbol{e}_{\Lambda=0}=(0,0,1)$ and $\boldsymbol{e}_{\Lambda= \pm 1}=(-1, \mp i, 0) / \sqrt{2}$ and $C$ is an overall constant which includes colour factors, the characteristic bag constants and the radial overlap integrals for the quark wavefunctions. The bag radius is $R_{B}$.

Our estimate of $C$ along the lines of [20], yields a relatively large value $C \sim 1.13$. The factor $\boldsymbol{e}_{\Lambda} \cdot\left(\boldsymbol{e}_{\Lambda_{1}}^{*} \wedge \boldsymbol{e}_{\Lambda_{2}}^{*}\right)$ is typical of the three-gluon vertex and is discussed in [19, 20]. 
One important result is that $\Delta M_{H}=0$ for $\Lambda=0$, i.e., for the $\Sigma$ state. The outcome can be summarized as:

$$
\begin{aligned}
\Delta M_{H} & =K c_{1} \alpha_{s} \times\left\{\begin{array}{ccc}
\Sigma & \Pi & J \\
0 & 1 & 2 \\
0 & -1 & 1 \\
0 & -2 & 0
\end{array}\right. \\
K & =\frac{1.13}{m_{b} R_{B}^{2}} .
\end{aligned}
$$

Our calculation is, by its very nature, approximate. For example, the bag is not spherical but elliptical, a correction included by Kuti and Morningstar 14] when calculating the $Q \bar{Q}$ BO potentials. Including this correction will increase the effect. Also, the bag radius is not fixed but varies with the quark motion in the $\mathrm{BO}$ approximation. The value we adopt is therefore associated with the most likely quark separation and we take $R_{B} \sim 1.5-2 \mathrm{GeV}^{-1}$. With $m_{b} \sim 4.75 \mathrm{GeV}$ we find $K \sim 60-110 \mathrm{MeV}$. With $c_{1}=1$ and $\alpha_{s}=0.4$ we find the scale of the induced spin-orbit splitting is $\sim 25-40 \mathrm{MeV}$ to be compared with $\sim 117 \mathrm{MeV}$ for the linear term in $c_{1}$ from eq(5.1). The value chosen for $\alpha_{s}$ is typical of bag calculations and is consistent with $\alpha_{V}(\mu)$ at a suitably chosen scale based on the B-quark mass [21]. While our prediction is too small our calculation is clearly incomplete. There are many other intermediate states that can contribute with similar magnitude of shift. The contributions of diagrams involving the interaction of the quarks with the constituent gluon are large partly because the colour factors are large and partly because there are many such terms. At $O\left(\alpha_{S}\right)$ there is a similar diagram to the one analyzed with $j=2$ electric gluon exchange. At $O\left(\alpha_{S}^{2}\right)$ there are diagrams involving the gluon four-point coupling, such as shown in Fig. 4, which has many orderings and gives contributions linear in $c_{1}$. There are many similar and other higher-order diagrams which contribute to the $c_{1}^{2}$ term. It cannot be excluded that there are also non-perturbative effects as well. The dependence of the induced mass shifts on the quantum numbers will be generally more complex than the $\boldsymbol{L} \cdot \mathbf{s}$ derived explicitly above, which nevertheless gives a qualitative picture of the pattern observed. We are therefore confident that our calculation sets a lower bound on the effect.

In the previous section we remarked that the $2^{-+}$rises above the $c_{1}=0$ reference mass by an amount smaller in magnitude than the shift of either the $0^{-+}$or $1^{-+}$below this point. This could be due to a cancellation between the linear and quadratic terms in eq(5.1). However, our operators do not distinguish between the $\Sigma$ and $\Pi$ states since they project out definite quark angular momentum $l_{Q \bar{Q}}=0$ which corresponds to an equally weighted mixture of $\Lambda=1,0,-1$. Based on the potentials of Kuti et. al [14] we see that the $\Sigma$ (which has the same $P C$ assignments as the $\Pi$ ) is about $150 \mathrm{MeV}$ above the $\Pi$. Since the $\Sigma$ is unaffected by the spin-orbit term it may be that we are seeing the $2^{-+} \Sigma$ state and not the $\Pi$ state because this latter has risen to a higher mass. We are testing this suggestion by constructing operators diagonal in $\Lambda$ and the results will be presented in a subsequent publication.

In order to confirm our observation that mixing with quarkonium states is negligible we have estimated the mixing in the BO approximation in the bag model. Since both source and sink contain a gluon field the gluon must be annihilated and subsequently recreated. A diagram of the kind that is relevant is shown in Fig. 5. The states that mix must have opposite spin and so the mixing is by a spin-flip operator, in this case it is proportional to $\left(\boldsymbol{\sigma}_{Q}-\boldsymbol{\sigma}_{\bar{Q}}\right)$. We calculate this term for the $s=1$, ground-state, 
hybrids mixing with the $s=0$ quarkonium state. A term of this kind has been discussed earlier by Ono [22] and Le Yaouanc et. al [15]. Our result is compatible with theirs and we find that the mixing amplitude from this diagram is $\sim 10^{-4}$. Ono [22] gives $\sim 2 \times 10^{-4}$. The hybrid- $Q \bar{Q}$ mass difference in lattice units is $\sim 0.36$ and so the effect of mixing at $t=10$ is $\sim 10^{-4} \times e^{-3.6} \sim .4 \%$. It is unlikely that mixing because of excited hybrids of the same quantum numbers will contribute appreciably and so we expect that mixing has negligible effect on the signal.

We believe that we have demonstrated that spin effects in heavy hybrid mesons are large and that the explanation is that the states expanded in the Fock basis contain more than one gluon constituent with an appreciable probability. Our partial estimate based on one intermediate state is $\sim 20-30 \%$ of the observed effect and shows that the order of magnitude can be explained. We are in no doubt that the complete sum over intermediate states will contribute to the shortfall and, because the effect at lowest non-zero order is so large, it is sensible to suppose that higher order corrections are appreciable. The most easily observed $1^{--}$state is unaffected since it has $s=0$ but indirect methods of production should eventually detect the $s=1$ states and then our observations can be tested.

\section{Discussion and Conclusions}

Ours is the first study on an anisotropic lattice that incorporates tuned Landau tadpoles in the action. The choice of Landau tadpoles was motivated by the observation that they yield better scaling behaviour for observables than plaquette tadpoles [8].

We have confirmed that the hybrids are at a levels in the range $900-1750 \mathrm{MeV}$ above the $1 S$ spin averaged ground state. This is consistent with our previous work and, allowing for systematic uncertainties in $a_{t}$ inevitable in a quenched calculation, is consistent with other evaluations.

The important new result of our calculation is that the effect of the spin interaction on the hybrid spectrum is unexpectedly large. We measure energy level splittings $\sim 100 \mathrm{MeV}$ and greater. The results are exhibited in Table 4 for a range of values of $c_{1}$ including, in some cases, unphysical negative values that help to establish parameters for the analytic form of the dependence on this coefficient.

In the absence of the spin interaction $\left(c_{1}=0\right)$, all the states we measure are degenerate. Because the exotic cannot mix with quarkonia, neither do the other states in the multiplet. When the spin interaction is switched on two effects can occur, (i) a shift of the energy levels of the hybrids and (ii) a mixing of the non-exotics with appropriate quarkonium states. We claim that our results are consistent with a scenario in which there is a direct effect on most of the energy levels but little mixing with quarkonia.

At tree level with no lattice artifact corrections, $c_{1}=1$. In deriving the NRQCD approximation from an interacting gauge theory we would expect corrections to $c_{1}$ of $O\left(\alpha_{s}\left(m a_{s}\right)^{2}\right)$. These could be large enough to yield $c_{1} \sim 2$. Hence, the spin effect we have simulated here may be a reasonable prediction even for $c_{1} \sim 2$. Because the terms in the NRQCD Hamiltonian which are $O\left(m v^{4}\right)$ and higher have neligible effect on the hybrid levels we can expect the effect of radiative corrections to be limited to a renormalization of $c_{1}$ which gives hope that the spin effects observed here are quantitatively correct.

It would be of great interest if any of these hybrid states could be observed although, 
except in the case of the $1^{--}$which can be directly produced in $e^{+} e^{-}$collisions, this would be experimentally difficult.

Future work will concentrate on using source and sink operators which have the quantum numbers of the Born-Oppenheimer approximation. This will allow us in particular to disentangle the $\Pi$ and $\Sigma$ states.

\section{References}

[1] C.T.H. Davies et al. Nucl. Phys. Proc. Suppl., 63:320-322, 1998. UKQCD collaboration.

[2] I.T. Drummond et al. Nucl. Phys. Proc. Suppl., 73:336-338, 1999.

[3] A. Ali Kahn et al. Submitted to Proceedings of LATTICE 99, hep-lat/9911039, 1999. CP-PACS Collaboration.

[4] M. Alford, T.R. Klassen, and G.P. Lepage. Phys. Rev., D58:034503, 1998.

[5] G.P. Lepage. Nucl. Phys. Proc. Suppl., 60A:267-278, 1998. International Workshop on Lattice QCD on Parallel Computers, Tsukuba.

[6] N.H. Shakespeare and H.D. Trottier. Phys. Rev., D59:014502, 1999.

[7] N.H. Shakespeare and H.D. Trottier. Nucl. Phys. Proc. Suppl., 73:342-344, 1999. Proceedings of LATTICE 98.

[8] M. Alford et al. Measuring the aspect ratio renormalisation of anisotropic-lattice gluons. 1999. preprint in preparation.

[9] T. Manke et al. Phys. Rev., D57:3829-3832, 1998. UKQCD Collaboration.

[10] T. Manke et al. Nucl. Phys. Proc. Suppl., 63:332-334, 1998. UKQCD Collaboration, Proceedings of LATTICE 97.

[11] M. Alford et al. Phys. Rev., D58:034503, 1998.

[12] P. Hasenfratz et al. Phys. Lett., B95:299-305, 1980.

[13] T. Manke et al. Phys. Rev. Lett., 82:4396-4399, 1999. CP-PACS Collaboration.

[14] K.J. Juge et al. Phys. Rev. Lett., 82:4400-4403, 1999.

[15] A. Le Yaouanc et al. Z. Phys. C., 28:309-315, 1985.

[16] P. Rakow. Private comminucation.

[17] F.E. Close and R.R. Horgan. Nucl. Phys., B164:413-426, 1980.

[18] F.E. Close and R.R. Horgan. Nucl. Phys., B185:333-345, 1981.

[19] T. Barnes et al. Nucl. Phys., B198:380-406, 1982.

[20] T. Barnes et al. Nucl. Phys., B224:241-264, 1983.

[21] A.M. Badalain and V.L. Morgunov. Phys. Rev., D60:116008, 1999.

[22] S. Ono. Z. Phys. C., 26:307, 1985. 


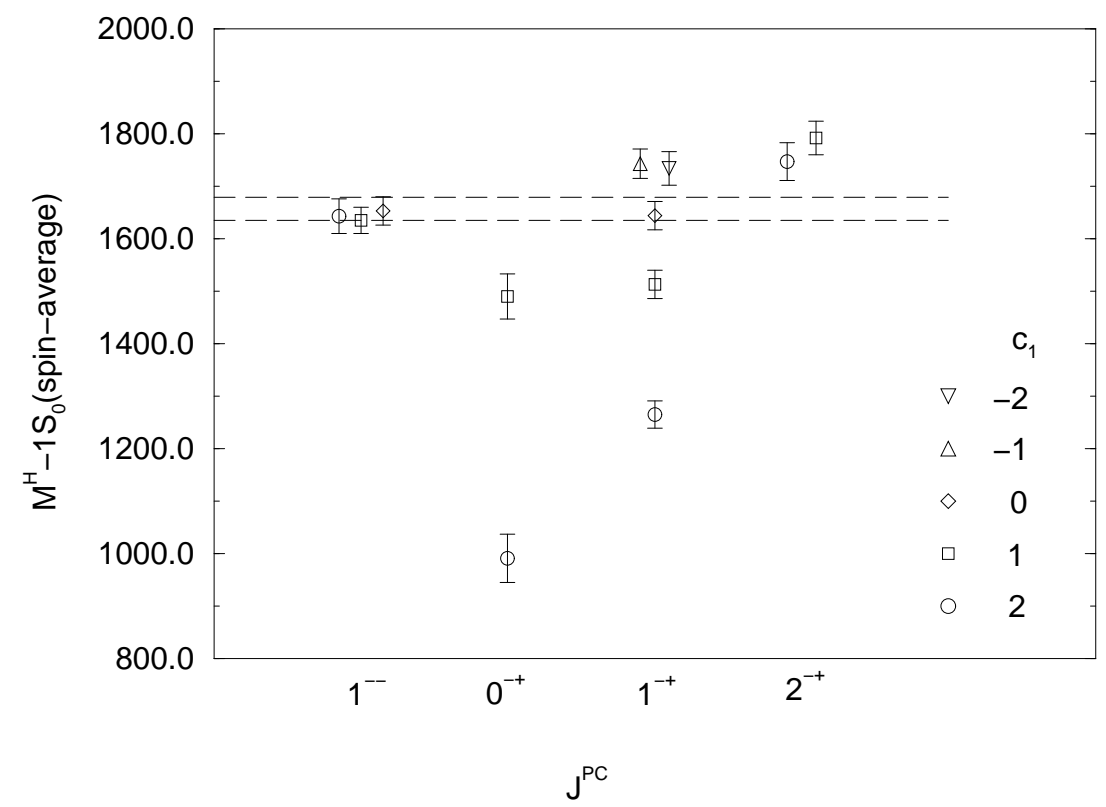

Figure 1: The variation of the hybrid masses with $c_{1}$ for the $1^{--}, 0^{-+}, 1^{-+}, 2^{-+}$.

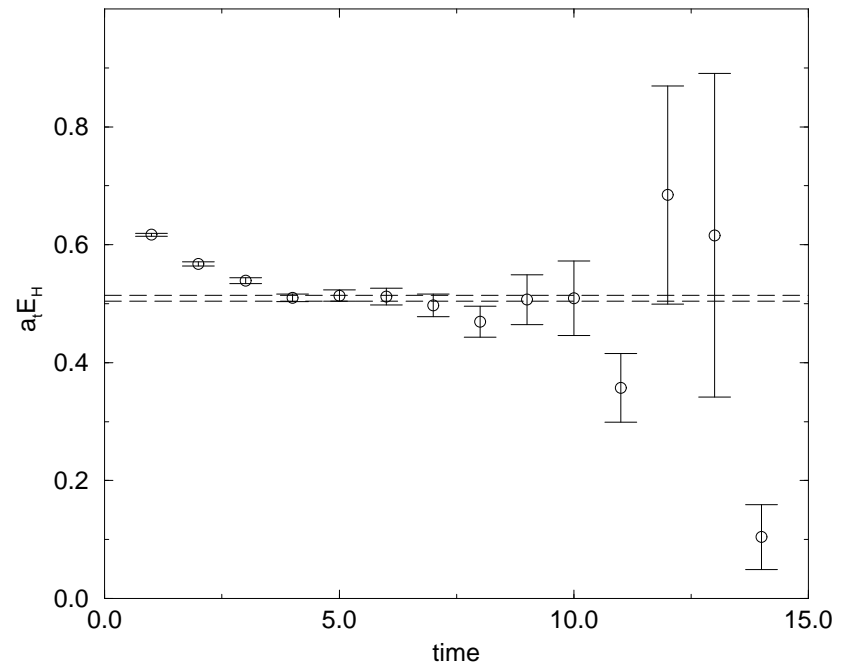

Figure 2: Effective mass plot for the $1^{-+}$hybrid with $c_{1}=-1$. The dashed lines show the upper and lower bounds of the fit. The plateau extends from $t=4-10$. 


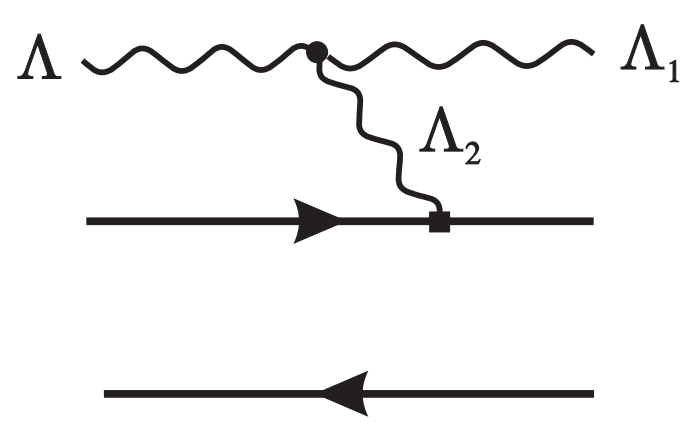

Figure 3: A representative graph for the second-order perturbation theory calculation discussed in section 6 for the mass shift due to the inclusion of the $\boldsymbol{\sigma} \cdot \boldsymbol{B}$ term in the NRQCD Hamiltonian (1.2). This spin operator is represented by the filled box and terms in the spin independent part of the interaction Hamiltonian are represented by filled circles. This contribution is to the linear term in $c_{1}$ in eq(5.1) and is $O\left(\alpha_{S}\right)$.

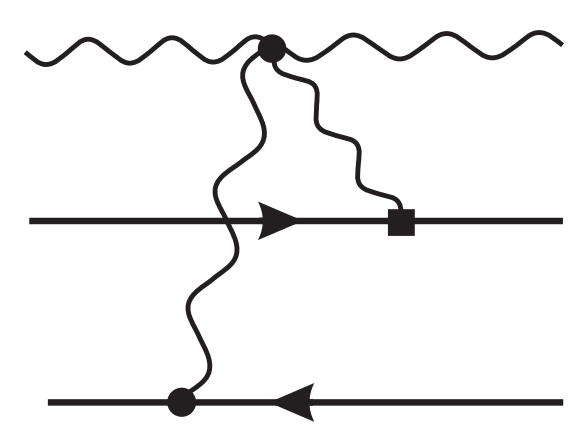

Figure 4: A higher-order, $O\left(\alpha_{S}^{2}\right)$, perturbation contribution to the linear term in $c_{1}$ in eq(5.1). Symbolism is the same as in Fig. 3 .

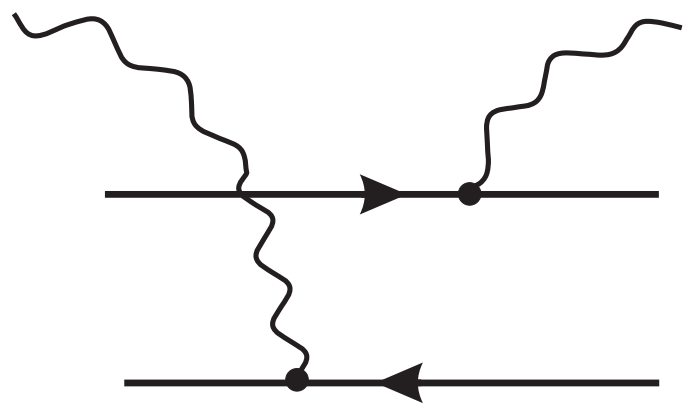

Figure 5: A representative graph for the mixing contribution to the hybrid-hybrid correlator from quarkonium intermediate states. 